\title{
SYNTHESIS AND In vitro ANTIMICROBIAL ACTIVITY OF SOME TRIAZOLE DERIVATIVES
}

\author{
RAVINESH MISHRA*, RAJIV KUMAR, SURESH KUMAR, JASEELA MAJEED, \\ MOHD RASHID, SAMEER SHARMA
}

"Department of Pharmaceutical Chemistry, Faculty of Pharmacy, Hamdard University, New Delhi 110 062, India.

(Received: December 21, 2009 - Accepted: July 13, 2010)

\begin{abstract}
Some 4-[\{1-(substituted)methylidine $\}$-amino]-3-(4-pyridyl)-5-mercapto-4H-1,2,4-triazol (3a- 3f) and $N$-[5-(4-substituted)-1H-1,2,3-triazol-1-yl]isonicotinamide derivatives (5a-5e) were synthesized by a sequence of reactions starting from isonicotinic acid hydrazide and is illustrated in scheme 1 and 2 . The antibacterial and antifungal activities of newly synthesized compounds were tested by the disc diffusion method using nutrient agar medium against various microorganisms such as gram positive Staphylococcus aureus and Bacillus subtilis, gram negative Escherichia coli and the fungi Aspergillus niger and Candida albicans. Ciprofloxacin and Fluconazole at $50 \mu \mathrm{g} / \mathrm{mL}$ were used as standard drugs for antibacterial and antifungal activities, respectively. All the synthesized compounds showed significant activity against various microorganisms.
\end{abstract}

Key words: Triazole derivatives, Antimicrobial activity, Ciprofloxacin, Fluconazole.

\section{INTRODUCTION}

Since the past few decades, the literature has been enriched with progressive findings about the synthesis and pharmacological activities of various substituted azole derivatives. It reveals that 1,2,4 and 1,2,3-triazoles possess broad spectrum of activities such as antimicrobial ${ }^{1-4}$, anti-inflammatory ${ }^{5}$, analgesic $^{6}$, antitumorial ${ }^{7}$, antihypertensives ${ }^{8}$, anticonvulsant and antiviral ${ }^{9}$. Some of the antimicrobial triazole derivatives used as common antibiotics such as Amphotericin B possess toxic effect on humans along with their antimicrobial effects ${ }^{10}$. Although there are antimicrobial agents having different structures are frequently used in the treatment of fungal infections, so there is increasing resistance to these drugs. To overcome the development of drug resistance, it is necessary to synthesize a new class of antimicrobial compounds possessing different chemical properties from those that are used commonly. 5-Thioxo1,2,4-triazole containing a substituted aromatic aldehyde side chain is a ideal heterocyclic for antifungal activity. The following antifungal 1,2,4-triazole derivatives are applicable in medicine like Fluconazole, Itraconazole and Terconazole ${ }^{11}$.

\section{EXPERIMENTAL}

The chemical reactives were supplied by E. Merck (Germany) and S.D Fine chemicals (India). Melting points were determined by open tube capillary method and were uncorrected. The purity of the compounds was checked on thin layer chromatography (TLC) plates (silica gel G) in toluene-ethyl formateformic acid (5:4:1) and benzene-methanol (8:2) solvent systems, the spots were located under iodine vapors and UV light. IR spectra were obtained on a Perkin-Elmer 1720 FT-IR spectrometer (KBr pellets). ${ }^{1} \mathrm{H}-\mathrm{NMR}$ spectra were recorded by a Bruker AC $300 \mathrm{MHz}$ spectrometer using TMS as internal standard in DMSO- $\mathrm{d}_{6} / \mathrm{CDCl}_{3}$ and mass spectra under electron impact conditions (EI) were recorded at $70 \mathrm{ev}$ ionizing voltage with a VG Prospec instrument and are presented as m/z. UV spectra were recorded on a UV-Visible Spectrophotometer Pharma Spec-1700 (SHIMADZU).

Synthesis of 4-amino-3-(4-pyridyl)-5-mercapto-4H-1,2,4-triazol (2)

Isonicotinic acid hydrazide $13.7 \mathrm{~g}(0.1 \mathrm{~mol})$ was dissolved in $200 \mathrm{~mL}$ absolute alcohol containing potassium hydroxide $11.2 \mathrm{~g}(0.1 \mathrm{~mol})$ at room temperature $12.5 \mathrm{~mL}$ carbondisulfide was added in parts and was stirred for 16 hours at room temperature. $100 \mathrm{~mL}$ of diethyl ether was added and stirred for further 3 h. $10.3 \mathrm{~g}(0.1 \mathrm{~mol}, 99 \%)$ hydrazine hydrate was added gradually to the potassium dithiocarbazinate salt dissolved in $100 \mathrm{~mL}$ water with stirring and was refluxed for $8 \mathrm{~h}$ during which hydrogen sulphide gas evolved and the colour of the reaction mixture changed to deep green. It was then cooled and acidified with hydrochloric acid to $\mathrm{pH} 1$. The yellow colored solid was isolated by filtration and recrystallised from ethanol to give compound (2) ${ }^{12}$.

General procedure for synthesis of 4 -[\{1-(substituted)methylidine $\}$ amino]-3-(4-pyridyl)-5-mercapto-4H-1,2,4-triazol derivatives (3a- 3f)

Few drops of glacial acetic acid were added to a solution of $0.01 \mathrm{~mol}$ of compound 2 (4-amino-3-(4-pyridyl)-5-mercapto-4H-1,2,4-triazol) in dimethylformamide $(20 \mathrm{~mL})$ and $0.01 \mathrm{~mol}$ of various benzaldehyde derivatives was added and refluxed for 9 hours. The reaction mixture was cooled and the pre- cipitate obtained was filtered, dried in vacuum and recrystallised from ethanol to give compound $\mathbf{3 a - 3 f}$. The compounds were synthesized as illustrated in scheme $1^{13}$.

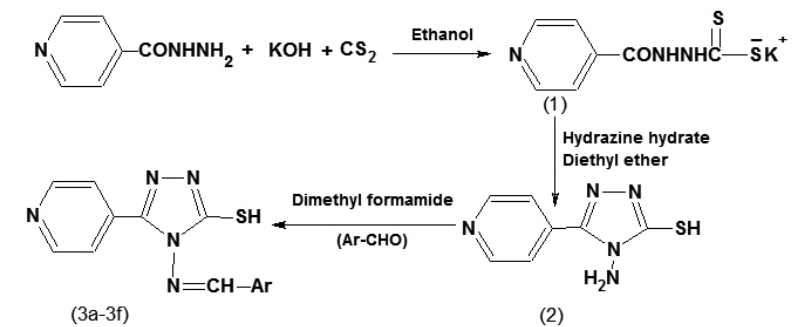

$\mathrm{Ar}=-\mathrm{C}_{6} \mathrm{H}_{5}-4 \mathrm{Cl}(3 \mathrm{a}),-\mathrm{C}_{6} \mathrm{H}_{5}-4 \mathrm{~N}\left(\mathrm{CH}_{3}\right)_{2}(3 \mathrm{~b}),-\mathrm{C}_{6} \mathrm{H}_{5}-3 \mathrm{NO}_{2}(3 \mathrm{c}),-\mathrm{C}_{6} \mathrm{H}_{5}-3 \mathrm{OH}(3 \mathrm{~d}),-\mathrm{C}_{6} \mathrm{H}_{5}-4 \mathrm{OCH} \mathrm{CH}_{3}(3 \mathrm{e}),-\mathrm{C}_{6} \mathrm{H}_{5}-3 \mathrm{Cl}(3 \mathrm{f})$

Scheme 1. 4-[\{1-(substituted)methylidine $\}$-amino]-3-(4-pyridyl)-5-mercapto-4H-1,2,4-triazol derivatives (3a-e).

General procedure for synthesis of $\mathrm{N}$-[5-(4-substituted)-1H-1,2,3-triazol-1-yl]isonicotinamide derivatives $(5 \mathrm{a}-5 \mathrm{e})$

Isonicotinic acid hydrazide $1.37 \mathrm{~g}(0.01 \mathrm{~mol})$ was dissolved in $30 \mathrm{~mL}$ ethanol and reacted with various equimolar substituted benzaldehyde derivatives were added in presence of few drops of glacial acetic acid and refluxed for $5 \mathrm{~h}$. Then it was cooled, filtered and recrystallised from ethanol. This gave an intermediate hydrazone compound, which was cyclised by passing freshly prepared diazomethane gas to the dissolved compound in 1,4 dioxane. The solution was allowed to stand for $24 \mathrm{~h}$ so that complete cyclisation took place, the product obtained after cooling was recrystallised from 1,4 dioxane to yield compound (5a-5e). The compounds were synthesized as is shown in scheme $2^{14,15}$.

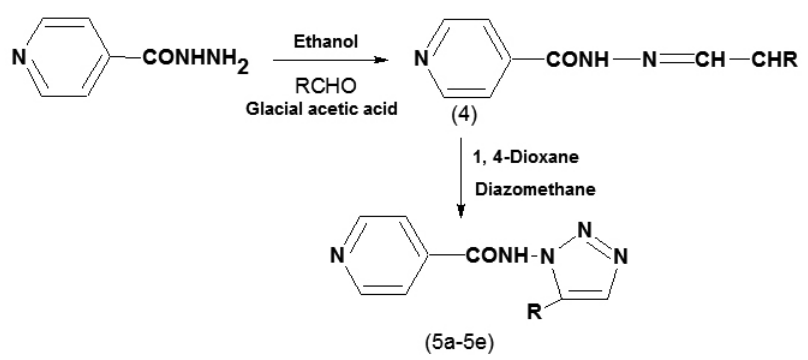

$\mathrm{R}=-\mathrm{C}_{6} \mathrm{H}_{5}-4 \mathrm{Cl}(5 \mathrm{a}),-\mathrm{C}_{6} \mathrm{H}_{5}-4 \mathrm{~N}\left(\mathrm{CH}_{3}\right)_{2}(\mathbf{5 b}),-\mathrm{C}_{6} \mathrm{H}_{5}-3,4-\left(\mathrm{OCH}_{3}\right)_{2}(5 \mathrm{c}),-\mathrm{C}_{6} \mathrm{H}_{5}-3-\mathrm{NO}_{2}(5 \mathrm{~d}),-\mathrm{C}_{6} \mathrm{H}_{5}-2-\mathrm{NO}_{2}(\mathbf{5 e})$

Scheme 2. N-[5-(4-substituted)-1H-1,2,3-triazol-1-yl]isonicotinamide derivatives $(5 \mathrm{a}-\mathrm{e})$. 


\section{Data Analysis}

4[\{1-(4'-chlorophenyl)methylidine\}amino]-3-(4-pyridyl)-5-mercapto4-1,2,4-triazole, (3a), colourless solid, yield $44 \%, \mathrm{mp} 276-278^{\circ} \mathrm{C}, \mathrm{R}_{\mathrm{f}} 0.76$, UV $\lambda$ (DMF) $245.8 \mathrm{~nm}$; FTIR (KBr) $v \mathrm{~cm}^{-1} 3013$ (Ar C-H stretching), 2563 (S-H stretching), 1603, 1485 ( $\mathrm{Ar} \mathrm{C}=\mathrm{C}$ stretching), 1563 (C=N stretching), 1313 (C-N stretching), 828 (C-Cl stretching), 688 (C-S stretching); ${ }^{1} \mathrm{H}$ NMR $(400 \mathrm{MHz}$ DMSO-d 6 ppm) $6.06(\mathrm{~s}, 1 \mathrm{H}, \mathrm{N}=\mathrm{CH}), 7.59-8.76(\mathrm{~m}, 8 \mathrm{H}$, aromatic protons), 12.59 (s, $1 \mathrm{H}, \mathrm{S}-\mathrm{H})$. Anal. calcd. for $\mathrm{C}_{14} \mathrm{H}_{10} \mathrm{~N}_{5} \mathrm{SCl}$ : C, 54.30; H, 4.25; $\mathrm{N}, 21.11$. Found: C, 54.34; H, 4.21; N, 21.09. FABMS (m/z) 314( $\left.\mathrm{M}^{+}\right), 315$ $\left(\mathrm{M}^{+}+1\right)$. Mol. Wt.:315.

4- [\{1-(4-dimethylaminophenyl) methylidine\}amino] -3 -(4-pyridyl)5-mercapto-4H-1,2,4-triazole, (3b), colourless solid, yield 31\%, mp 224 $226^{\circ} \mathrm{C}, \mathrm{R}_{\mathrm{f}} 0.82$, UV $\lambda \quad$ (DMF) $260.7 \mathrm{~nm}$; FTIR (KBr) $v \mathrm{~cm}^{-1} 3132$ (Ar C-H stretching), 2544 ( $\mathrm{S}-\mathrm{H}$ stretching ), $1523(\mathrm{C}=\mathrm{N}$ stretching), 1344 (C-N stretching), $671 \mathrm{~cm}^{-1}$ (C-S stretching); ${ }^{1} \mathrm{H}$ NMR (400 MHz DMSO-d $\delta$ ppm) $3.20[\mathrm{~s}$, $\left.6 \mathrm{H}, \mathrm{N}\left(\mathrm{CH}_{3}\right)_{2}\right], 8.08(\mathrm{~s}, 1 \mathrm{H}, \mathrm{N}=\mathrm{CH}), 7.59-8.78(\mathrm{~m}, 8 \mathrm{H}$, aromatic protons), 12.32 (s, $1 \mathrm{H}, \mathrm{SH})$. Anal. calcd. for $\mathrm{C}_{15} \mathrm{H}_{16} \mathrm{~N}_{6} \mathrm{~S}: \mathrm{C}, 57.67 ; \mathrm{H}, 5.16 ; \mathrm{N}, 26.90$. Found: $\mathrm{C}$, 57.64; H, 5.18; N, 26.93. FABMS (m/z) $323\left(\mathrm{M}^{+}\right), 324\left(\mathrm{M}^{+}+1\right)$. Mol. Wt.: 324.

4-[\{ 1-(3'-nitrophenyl)methylidine\}amino]-3-(4-pyridyl)-5-mercapto$4 \mathrm{H}$-1,2,4-triazol e, (3c), colourless solid, yield $66 \%, \mathrm{mp} 288-290^{\circ} \mathrm{C}, \mathrm{R}_{\mathrm{c}} 0.79$, UV $\lambda_{\max }$ (DMF) $221.9 \mathrm{~nm}$; FTIR (KBr) $v \mathrm{~cm}^{-1} 3033$ (Ar C-H stretching), 2579 (S-H stretching), 1609, 1585, 1452 (Ar $\mathrm{C}=\mathrm{C}$ stretching), $1520(\mathrm{C}=\mathrm{N}$ stretching), 1353 (Ar-NO $), 1315$ (C-N stretching), $698 \mathrm{~cm}^{-1}$ (C-S stretching); ${ }^{1} \mathrm{H}$ NMR $(400 \mathrm{MHz}$ DMSO-d $\delta$ ppm) $5.58(\mathrm{~s}, 1 \mathrm{H}, \mathrm{N}=\mathrm{CH}), 7.78-8.71(\mathrm{~m}, 8 \mathrm{H}$, aromatic protons), $14.02(\mathrm{~s}, 1 \mathrm{H}, \mathrm{S}-\mathrm{H})$. Anal. calcd. for $\mathrm{C}_{14} \mathrm{H}_{10} \mathrm{~N}_{6} \mathrm{SO}_{2}: \mathrm{C}, 51.53$; $\mathrm{H}, 3.09 ; \mathrm{N}, 25.02$. Found: C, 51.52; H, 3.12; N, 25.14. FABMS (m/z) 325( $\left.\mathrm{M}^{+}\right)$, 326( $\left.\mathrm{M}^{+}+1\right)$. Mol. Wt.: 326 .

4-[\{1-(3'-hydroxyphenyl)methylidine $\}$ amino]-3-(4-pyridyl) $\quad$-5-mercapto-4H-1,2,4-triazole, (3d), colourless solid, yield $62 \%, \mathrm{mp} 282-284^{\circ} \mathrm{C}$, $\mathrm{R}_{\mathrm{f}} 0.66$, UV $\lambda_{\text {max }}$ (DMF) $234.6 \mathrm{~nm}$; FTIR (KBr) $v \mathrm{~cm}^{-1} 3450$ (O-H stretching), 3032 (Ar C-H stretching), 2579 (S-H stretching), 1609, 1585, 1453 (Ar C=C stretching), 1546 (C=N stretching), 1315 (C-N stretching), 699 (C-S stretching); ${ }^{1} \mathrm{H}$ NMR (400 MHz DMSO-d $\delta$ ppm) $5.64(\mathrm{~s}, 1 \mathrm{H}, \mathrm{N}=\mathrm{CH}), 7.82(\mathrm{~s}, 1 \mathrm{H}$ $\mathrm{O}-\mathrm{H}), 7.86-8.72(\mathrm{~m}, 8 \mathrm{H}$, aromatic protons), $13.96(\mathrm{~s}, 1 \mathrm{H}, \mathrm{S}-\mathrm{H})$. Anal. calcd. for $\mathrm{C}_{14} \mathrm{H}_{11} \mathrm{~N}_{5} \mathrm{SO}$ : C, 56.55; H, 3.73; N, 23.10. Found: C, 56.58; H, 3.76; N, 23.13. FABMS (m/z) 296(M+), 297(M+1). Mol. Wt.: 297.

4-[\{1-(4'-methoxyphenyl)methylidine $\}$ amino]-3-(4-pyridyl)-5-mercapto-4H-1,2,4-triazole, (3e), colourless solid, yield $50.8 \%, \mathrm{mp} 272-274^{\circ} \mathrm{C}$, $\mathrm{R}_{\mathrm{f}} 0.72$, UV $\lambda$ (DMF) $212.4 \mathrm{~nm}$; FTIR (KBr) $v \mathrm{~cm}^{-1} 3051$ (Ar C-H stretching), $2872(\mathrm{C}-\mathrm{O}-\mathrm{CH}$ ), 2621 (S-H stretching), 1606 ( $\mathrm{Ar} \mathrm{C}=\mathrm{C}$ stretching), 1541 (C=N stretching), 1377 (C-N stretching), 678 (C-S stretching); ${ }^{1} \mathrm{H}$ NMR (400 MHz DMSO-d $\delta$ ppm) 3.79 (s, 3H, -O-CH $), 7.67(\mathrm{~s}, 1 \mathrm{H}, \mathrm{N}=\mathrm{CH}), 7.25-7.80$ (m, $8 \mathrm{H}$, aromatic protons), $11.02(\mathrm{~s}, 1 \mathrm{H}, \mathrm{SH})$. Anal. calcd. for $\mathrm{C}_{15} \mathrm{H}_{13} \mathrm{~N}_{5} \mathrm{SO}: \mathrm{C}$, 57.86; H, 4.21; N, 22.49. Found: C, 57.84; H, 4.22; N, 22.46. FABMS (m/z) $310\left(\mathrm{M}^{+}\right), 311\left(\mathrm{M}^{+}+1\right)$. Mol. Wt.: 311 .

4-[\{1-(3'-chlorophenyl)methylidine\}amino]-3-(4-pyridyl)-5-mercapto$4 \boldsymbol{H}-1,2,4-$ triazole, (3f), colourless solid, yield $51 \%$, mp $296-298^{\circ} \mathrm{C}, \mathrm{R}_{\mathrm{f}}$ : 0.81 , UV $\lambda$ (DMF) $240.6 \mathrm{~nm}$; FTIR (KBr) $v \mathrm{~cm}^{-1} 2975$ (Ar C-H stretching), 2648 ( $\mathrm{m}-\mathrm{H}$ stretching), 1664 ( $\mathrm{Ar} \mathrm{C}=\mathrm{C}), 1523$ ( $\mathrm{C}=\mathrm{N}$ stretching), 1365 (C-N stretching), 815 (C-Cl stretching), 682 (C-S stretching). ${ }^{1} \mathrm{H}$ NMR (400 MHz DMSO-d $\delta$ ppm) $5.69(\mathrm{~s}, 1 \mathrm{H}, \mathrm{N}=\mathrm{CH}), 7.72-7.90(\mathrm{~m}, 4 \mathrm{H}$, Aromatic protons) $8.01-8.13(\mathrm{~m}, 4 \mathrm{H}$, aromatic protons), $12.49(\mathrm{~s}, 1 \mathrm{H}, \mathrm{S}-\mathrm{H})$. Anal. calcd. for $\mathrm{C}_{14} \mathrm{H}_{10} \mathrm{~N}_{5} \mathrm{SC}: \mathrm{C}, 53.25 ; \mathrm{H}, 3.19 ; \mathrm{N}, 22.18$. Found: C, 53.29; H, 3.17; N, 22.20. FABMS (m/z) 314( $\left.\mathrm{M}^{+}+1\right), 315\left(\mathrm{M}^{+}+1\right)$. Mol. Wt.: 315.

$\mathrm{N}$-[5-(4'-chlorophenyl)-1H-1,2,3-triazol 1-yl]isonicotinamide, (5a), colourless solid, yield $70.01 \%$, mp $202-204^{\circ} \mathrm{C}, \mathrm{R}_{\mathrm{f}} 0.73$, UV $\lambda_{\text {max }}$ (DMF) 310.6 $\mathrm{nm}$; FTIR (KBr) $v \mathrm{~cm}^{-1} 3428$ (N-H stretching), 3067 (Ar C-H stretching), $1664(\mathrm{C}=\mathrm{O}$ stretching $), 1550(\mathrm{C}=\mathrm{N}), 1334(\mathrm{C}-\mathrm{N}$ stretching $), 818(\mathrm{C}-\mathrm{Cl}$ stretching); ${ }^{1} \mathrm{H}$ NMR (400 MHz DMSO-d 6 ' $\delta$ ppm) 5.32 (s, $1 \mathrm{H}, \mathrm{CH}$ of triazole), 7.64$7.85(\mathrm{~m}, 6 \mathrm{H}$, aromatic protons $), 8.06(\mathrm{~m}, 2 \mathrm{H}$, aromatic protons $), 11.83(\mathrm{~s}, 1 \mathrm{H}$, $\mathrm{CONH}$ ). Anal. calcd for $\mathrm{C}_{14} \mathrm{H}_{10} \mathrm{~N}_{5} \mathrm{OCl}$ : C, 60.83; $\mathrm{H}, 3.53 ; \mathrm{N}, 21.83$. Found: $\mathrm{C}$, 60.80; H, 3.54; N, 21.84. FABMS (m/z) $298\left(\mathrm{M}^{+}\right), 299\left(\mathrm{M}^{+}+1\right)$. Mol. Wt.: 299.

$\mathrm{N}$-[5-(4-dimethylaminophenyl)-1H-1,2,3-triazol-1-yl]isonicotinamide, (5b), colourless solid, yield $77 \%, \mathrm{mp} 196-198^{\circ} \mathrm{C}, \mathrm{R}_{\mathrm{f}} 0.77$, UV $\lambda$ (DMF) $363.2 \mathrm{~nm}$; FTIR (KBr) $v \mathrm{~cm}^{-1}$ 3421(N-H stretching), 3067 (Ar C-H), 1667 $\left(\mathrm{C}=\mathrm{O}\right.$ stretching), $1597(\mathrm{C}=\mathrm{N}$ stretching $), 1393(\mathrm{C}-\mathrm{N}$ of triazole $) ;{ }^{1} \mathrm{H}$ NMR $(400$ $\mathrm{MHz}$ DMSO-d $\delta$ ppm) $3.20\left(\mathrm{~s}, 6 \mathrm{H}, \mathrm{N}\left(\mathrm{CH}_{3}\right)_{2}\right), 5.58(\mathrm{~s}, 1 \mathrm{H}, \mathrm{C}-\mathrm{H}$ of triazole $)$, 7.59-9.28 ( $\mathrm{m}, 8 \mathrm{H}$, aromatic protons), $11.67(\mathrm{~s}, 1 \mathrm{H}, \mathrm{CONH})$. Anal. calcd. for $\mathrm{C}_{16} \mathrm{H}{ }_{10} \mathrm{O}: \mathrm{C}, 67.90 ; \mathrm{H}, 5.70 ; \mathrm{N}, 26.24$. Found: $\mathrm{C}, 67.91 ; \mathrm{H}, 5.73 ; \mathrm{N}, 26.39$. FABMS $(\mathrm{m} / \mathrm{z}) 307\left(\mathrm{M}^{+}\right), 308\left(\mathrm{M}^{+}+1\right)$. Mol. Wt.:308

$\mathrm{N}$-[5-(3',4'-dimethoxyphenyl)-1 H-1,2,3-triazol-1-yl]isonicotinamide, (5c), colourless solid, yield $68 \%, \mathrm{mp} 172^{\circ} \mathrm{C}, \mathrm{R}_{\mathrm{f}} 0.77$, UV $\lambda_{\max }$ (DMF) 363.2 $\mathrm{nm}$; FTIR (KBr) $v \mathrm{~cm}^{-1} 3428$ (N-H stretching), 3044 (Ar C-H stretching), 2835
(C-O-CH ${ }_{3}$ stretching), $1644(\mathrm{C}=\mathrm{O}$ stretching $), 1598(\mathrm{C}=\mathrm{N}$ stretching $), 1371$ (C-N stretching); ${ }^{1} \mathrm{H}$ NMR (400 MHz DMSO-d 6 $\delta$ ppm) $3.93\left[\mathrm{~s}, 6 \mathrm{H},\left(\mathrm{CH}_{3}\right)_{2}\right]$, 7.10 (s, $1 \mathrm{H}, \mathrm{C}-\mathrm{H}$ of triazole), $7.68-8.77(\mathrm{~m}, 7 \mathrm{H}$, aromatic protons), 10.45 (s, $1 \mathrm{H}, \mathrm{CONH}$ ); Anal. calcd. for $\mathrm{C}_{1} \mathrm{H}_{9} \mathrm{~N}_{5} \mathrm{O}: \mathrm{C}, 63.82 ; \mathrm{H}, 5.00 ; \mathrm{N}, 19.85$. Found: C, 63.79; H, 5.04; N, 19.83. FABMS (m/z) 324( $\left.\mathrm{M}^{+}\right)$, 325( $\left.\mathrm{M}^{+}+1\right)$. Mol. Wt.: 325.

$\mathrm{N}$-[5-(3'-nitrophenyl)-1H-1,2,3-triazol-1-yl]isonicotinamide, (5d), colourless solid, yield $69 \%, \mathrm{mp} 222-224^{\circ} \mathrm{C}, \mathrm{R}_{\mathrm{f}} 0.83$, UV $\lambda$ (DMF) $292.6 \mathrm{~nm}$; FT IR $(\mathrm{KBr}) v \mathrm{~cm}^{-1} 3439(\mathrm{NH}$ stretching of CONH), $3009(\mathrm{Ar} \mathrm{C}-\mathrm{H}), 1678$ $(\mathrm{C}=\mathrm{O}$ stretching $), 1560(\mathrm{C}=\mathrm{N}), 1516\left(\mathrm{NO}_{2}\right.$ stretching $) ;{ }^{1} \mathrm{H}$ NMR $(400 \mathrm{MHz}$ DMSO-d $\left.{ }_{6} \delta \mathrm{ppm}\right) 5.56(\mathrm{~s}, 1 \mathrm{H}, \mathrm{CH}$ of triazole), 7.59-9.28 (m, $8 \mathrm{H}$, aromatic protons), $12.30(\mathrm{~s}, 1 \mathrm{H}, \mathrm{CONH})$. Anal. calcd for $\mathrm{C}_{14} \mathrm{H}_{10} \mathrm{~N}_{5} \mathrm{O}_{3}: \mathrm{C}, 58.43 ; \mathrm{H}, 3.39 ; \mathrm{N}$, 26.21. Found: C, 58.47; H, 3.34; N, 26.25. FABMS (m/z) 309( $\left.\mathrm{M}^{+}\right), 310\left(\mathrm{M}^{+}+1\right)$. Mol. Wt.: 310 .

$\mathrm{N}$-[5-(2'-nitrophenyl)-1H-1,2,3-triazol-1-yl]isonicotinamide, (5e), colourless solid, yield $81 \%, \mathrm{mp} 218-220^{\circ} \mathrm{C} ; \mathrm{R}_{\mathrm{f}}: 0.77$, UV $\lambda_{\text {max }}$ (DMF) $288.7 \mathrm{~nm}$. FTIR (KBr) $v \mathrm{~cm}^{-1} 3441$ (NH stretching of CONH), 3019 (Ar C-H stretching), $1681\left(\mathrm{C}=\mathrm{O}\right.$ stretching), $1564 \quad\left(\mathrm{C}=\mathrm{N}\right.$ stretching), 1512 ( $\mathrm{NO}_{2}$ stretching), 1348 (C-N stretching); ${ }^{1} \mathrm{H}$ NMR (400 MHz DMSO-d 6 ppm) $5.85(\mathrm{~s}, 1 \mathrm{H}, \mathrm{CH}$ of triazole), 7.54-7.80 (m, 8H, Ar C-H), $11.02(\mathrm{~s}, 1 \mathrm{H}, \mathrm{CONH})$. Anal. calcd for $\mathrm{C}_{14} \mathrm{H}_{10} \mathrm{~N}_{5} \mathrm{O}_{3}: \mathrm{C}, 58.43 ; \mathrm{H}, 3.39 ; \mathrm{N}, 26.21$. Found: C, 58.40; H, 3.35; N, 26.24. FABMS (m/z) $309\left(\mathrm{M}^{+}\right), 310\left(\mathrm{M}^{+}+1\right)$. Mol. Wt.: 310.

\section{In vitro antibacterial activity}

The primary screen was carried out using the agar disc-diffusion method ${ }^{16}$ using Muller-Hinton agar medium. Sterile filter paper discs moistened with the test compound solution in dimethylsulfoxide at concentration of $200 \mu \mathrm{g} /$ $\mathrm{mL}$ were carefully placed on the agar cultures plates that had been previously inoculated separately with the microorganisms ${ }^{17,18}$. The plates were incubated at $37^{\circ} \mathrm{C}$. The synthesized compounds were tested for their in vitro antimicrobial activity against the Gram-positive bacteria S. aureus ATCC 19433 and B. subtilis ATCC 6633, the Gram-negative bacteria E. coli ATCC 25922, and the filamentous fungus $A$. niger ATCC 1034 and yeast-like pathogenic fungus C. albicans ATCC 753. The standards, Ciprofloxacin and Fluconazole were dissolved in dimethylsulfoxide at concentration of $800 \mu \mathrm{g} / \mathrm{mL}$. The microorganism suspensions at $10^{6} \mathrm{CFU} / \mathrm{mL}$ (colony forming unit $/ \mathrm{mL}$ ) concentrations were inoculated to the corresponding wells. The Petri plates were incubated at $36^{\circ} \mathrm{C}$ for 24 and $48 \mathrm{~h}$ for the bacteria and fungi, respectively. The MIC values were determined as the lowest concentration that completely inhibited visible growth of the microorganism as detected by unaided eye. The diameters of the growth inhibition zones were measured after $24 \mathrm{~h}$ for all microorganisms but after $48 \mathrm{~h}$ in case of $C$. albicans.

\section{Preparation of agar wells}

A variety of devices such as stainless-steel or ceramic cylinders, single or multiple in design, and paper discs of several sizes are commonly used for antimicrobial assays performed by the agar diffusion method. Holes cut in agar (agar wells) are occasionally useful for the assay of such materials as serum, milk, eggs, or viscous materials. The routine use of agar wells is hampered by the tedium of cutting and removing the agar plugs without disrupting the agar layer ${ }^{19}$. Sealing the wells with melted agar is often necessary to obtain uniform inhibition zones ${ }^{20}$. Agar wells are cut from seeded agar plates by a cylindrical metal tube attached through a trap to a vacuum source. A section of a no. 4 cork borer is a convenient cutter. A most important addition is a $1.5 \mathrm{~mm}$ diameter hole in the side of the cutter, about $10 \mathrm{~mm}$ from the cutting edge, which acts as a vacuum bypass and prevents tearing the agar from the bottom of the plate. The agar plate is placed in the template on which are marked six evenly spaced locations for the agar wells. The operator cuts the agar with rapid strokes, holding the cutter vertical to assure even contact with the base of the plate. Cutting is greatly simplified if the plates are refrigerated at least $30 \mathrm{~min}$ prior to cutting. The firmness of cold agar is very helpful. An agar layer 5 to $6 \mathrm{~mm}$ thick makes convenient-size wells.

\section{RESULTS AND DISCUSSION}

\section{Chemistry}

The synthesis of compounds $3 \mathrm{a}-\mathrm{f}$ and $5 \mathrm{a}-\mathrm{e}$ were undertaken as per the scheme 1 and 2 . The required 4-amino-3-(4-pyridyl)-5-mercapto-4H-1,2,4-triazol (2) was prepared by the action of isonicotinic acid hydrazide and carbodisulfide. The various 4-[\{1-(substituted)methylidine $\}$-amino]-3-(4-pyridyl)-5mercapto-4H-1,2,4-triazol derivatives (3a-f) were synthesized by condensation of 4-amino-5-(pyridin-4-yl)-4H-1,2,4-triazole-3-thiol with various aromatic aldehydes in dimethyl formamide, in a yield ranging between 31 to $68 \%$. Some $N$-[5-(4-substituted)-1H-1,2,3-triazol-1-yl]isonicotinamide derivatives (5a-e) were synthesized by a sequence of reactions starting from isonicotinic acid 
hydrazide with aromatic aldehydes in glacial acetic acid. ${ }^{1} \mathrm{H}-\mathrm{NMR}$, Mass and IR spectra were recorded on Bruker DRX-300 (300 MHz), MS Jeol SX-102 (FAB) and BIORAD FTIR Spectrometer instruments respectively. The elemental analysis data of compounds was found to be within $\pm 0.4 \%$ limit. The IR spectra of final compounds (3a-f) and (5a-e) showed absorption bands at 2544-2648 $\mathrm{cm}^{-1}$ and $1644-1681 \mathrm{~cm}^{-1}$ appearing due to the presence of a $\mathrm{C}=\mathrm{O}$ functional group and a $\mathrm{SH}$ functional group respectively, while the band observed at $1516-1598 \mathrm{~cm}^{-1}$ corresponds to $\mathrm{C}=\mathrm{N}$ linkage. Through the $1 \mathrm{H}-\mathrm{NMR}$ spectra the synthesis of the compounds (3a-f) was confirmed on the basis that -SH proton which was observed as singlet at $\delta 11.02-\delta 14.02$. The CONH proton resonated in compounds (5a-e) at $\delta$ values between 11.02-14.02 as singlet to broad singlet and this signal was absent when $1 \mathrm{H}-\mathrm{NMR}$ was recorded in the presence of $\mathrm{D}_{2} \mathrm{O}$.

\section{Antimicrobial activity}

The results revealed that majority of the synthesized compounds showed varying degrees of inhibition against the tested microorganisms. In general, the inhibitory activity against the tested gram-positive bacteria was higher than that of the gram-negative bacteria, and some derivatives showed moderate or weak activity against $C$. albicans and A. niger. The results indicated that the longer the side chain of a compound, the more antimicrobial activity it possesses. Among the benzene ring ortho-substitution series, methyl derivatives are more active than the hydroxyl and methoxyl substituted derivatives. However, all the tested compounds exhibited low antifungal activity against $C$. albicans. Moreover, the compounds $\mathbf{3} \mathbf{b}, \mathbf{5 a}, \mathbf{5 b}$ having the side chain showed higher activity than oxime-ether derivatives with the same side chain against S. aureus. This bioisosteric replacement resulted in a slightly increased antimicrobial activity. Our study revealed that all the compounds had stronger antibacterial activity against Gram positive bacteria when compared to Gram negative bacteria. The reason for the weaker antifungal activity according to antibacterial effect might be postulated as different action in the mechanism of the compounds such as inhibition effect on respiratory systems of fungal cells, rather than cell wall destruction. The antimicrobial activity revealed that newly synthesized compound $\mathbf{3 b} \mathbf{b} \mathbf{5}$ and $\mathbf{5 b}$ showed good significant activity. The results of the preliminary antimicrobial testing of the prepared compounds, the typical broad spectrum antibacterial drug Ciprofloxacin $(50 \mu \mathrm{g} / \mathrm{mL})$ and the potent antifungal drug Fluconazole $(50 \mu \mathrm{g} / \mathrm{mL})$ are shown in Table 1 and 2.

Table 1- Antibacterial activity of compounds.

\begin{tabular}{|c|c|c|c|c|c|c|c|c|c|}
\hline \multirow[b]{2}{*}{ Compd. No. } & \multicolumn{9}{|c|}{ Diameter of zone of inhibition (mm) } \\
\hline & $25 \mathrm{mg} \mathrm{ml}^{-1}$ & $50 \mathrm{mg} \mathrm{ml}^{-1}$ & $100 \mathrm{mg} \mathrm{ml}^{-1}$ & $25 \mathrm{mg} \mathrm{ml}^{-1}$ & $50 \mathrm{mg} \mathrm{ml}^{-1}$ & $100 \mathrm{mg} \mathrm{ml}^{-1}$ & $25 \mathrm{mg} \mathrm{ml}^{-1}$ & $50 \mathrm{mg} \mathrm{ml}^{-1}$ & $\begin{array}{c}100 \mathrm{mg} \\
\mathrm{ml}^{-1}\end{array}$ \\
\hline 2 & $\begin{array}{c}4.93 \\
\pm 0.26\end{array}$ & $9.86 \pm 0.15$ & $\begin{array}{l}15.23 \\
\pm 0.15\end{array}$ & $\begin{array}{c}4.46 \\
\pm 0.30\end{array}$ & $8.8 \pm 0.20$ & $\begin{array}{l}14.36 \\
\pm 0.15\end{array}$ & $4.66 \pm 0.20$ & $10.76 \pm 0.15$ & $\begin{array}{c}16.6 \\
\pm 0.20\end{array}$ \\
\hline $3 \mathbf{b}$ & $\begin{array}{c}10.8 \\
\pm 0.20\end{array}$ & $\begin{array}{l}17.86 \\
\pm 0.12\end{array}$ & $\begin{array}{l}22.83 \\
\pm 0.15\end{array}$ & $\begin{array}{c}8.93 \\
\pm 0.15\end{array}$ & $\begin{array}{l}14.80 \\
\pm 0.15\end{array}$ & $20.26 \pm 0.15$ & $\begin{array}{c}9.4 \\
\pm 0.20\end{array}$ & $\begin{array}{c}16.4 \\
\pm 0.10\end{array}$ & $\begin{array}{c}21.3 \\
\pm 0.10\end{array}$ \\
\hline $3 \mathbf{c}$ & $\begin{array}{c}7.8 \\
\pm 0.20\end{array}$ & $\begin{array}{l}14.96 \\
\pm 0.20\end{array}$ & $\begin{array}{l}19.71 \\
\pm 0.20\end{array}$ & $\begin{array}{c}7.9 \\
\pm 0.10\end{array}$ & $\begin{array}{l}13.83 \\
\pm 0.25\end{array}$ & $\begin{array}{l}18.16 \\
\pm 0.20\end{array}$ & $8.40 \pm 0.10$ & $\begin{array}{l}14.63 \\
\pm 0.15\end{array}$ & $\begin{array}{l}19.96 \\
\pm 0.15\end{array}$ \\
\hline $3 e$ & $\begin{array}{l}11.66 \\
\pm 0.11\end{array}$ & $\begin{array}{l}18.26 \\
\pm 0.12\end{array}$ & $23.83 \pm 0.15$ & $\begin{array}{c}9.76 \\
\pm 0.15\end{array}$ & $\begin{array}{l}15.63 \\
\pm 0.20\end{array}$ & $\begin{array}{c}20.8 \\
\pm 0.10\end{array}$ & $\begin{array}{c}10.3 \\
\pm 0.10\end{array}$ & $\begin{array}{l}17.36 \\
\pm 0.15\end{array}$ & $\begin{array}{c}22.4 \\
\pm 0.20\end{array}$ \\
\hline $3 f$ & $\begin{array}{c}6.9 \\
\pm 0.10\end{array}$ & $12.93 \pm 0.15$ & $18.9 \pm 0.10$ & $\begin{array}{c}5.33 \\
\pm 0.56\end{array}$ & $\begin{array}{l}11.45 \\
\pm 0.37\end{array}$ & $\begin{array}{l}16.45 \\
\pm 0.00\end{array}$ & $6.71 \pm 0.26$ & $\begin{array}{l}12.56 \\
\pm 0.05\end{array}$ & $18.6 \pm 0.20$ \\
\hline $5 \mathbf{a}$ & $9.13 \pm 0.10$ & $\begin{array}{l}18.86 \\
\pm 0.12\end{array}$ & $22.8 \pm 0.20$ & $\begin{array}{l}10.33 \\
\pm 0.20\end{array}$ & $\begin{array}{c}14.7 \\
\pm 0.30\end{array}$ & $20.9 \pm 0.20$ & $\begin{array}{l}10.63 \\
\pm 0.15\end{array}$ & $\begin{array}{c}17.4 \\
\pm 0.20\end{array}$ & $23.7 \pm 0.10$ \\
\hline $5 b$ & $\begin{array}{c}10.9 \\
\pm 0.15\end{array}$ & $\begin{array}{c}19.7 \\
\pm 0.26\end{array}$ & $\begin{array}{c}23.3 \\
\pm 0.17\end{array}$ & $\begin{array}{c}10.2 \\
\pm 0.17\end{array}$ & $\begin{array}{l}15.16 \\
\pm 0.10\end{array}$ & $\begin{array}{l}21.16 \\
\pm 0.10\end{array}$ & $10.76 \pm 0.15$ & $\begin{array}{c}19.6 \\
\pm 0.20\end{array}$ & $\begin{array}{l}24.83 \\
\pm 0.15\end{array}$ \\
\hline $5 c$ & $\begin{array}{c}5.9 \\
\pm 0.10\end{array}$ & $\begin{array}{l}10.76 \\
\pm 0.15\end{array}$ & $\begin{array}{l}16.53 \\
\pm 0.12\end{array}$ & $\begin{array}{c}4.43 \\
\pm 0.15\end{array}$ & $\begin{array}{l}11.36 \\
\pm 0.21\end{array}$ & $\begin{array}{l}17.73 \\
\pm 0.25\end{array}$ & $\begin{array}{c}6.16 \\
\pm 0.15\end{array}$ & $\begin{array}{l}11.63 \\
\pm 0.15\end{array}$ & $\begin{array}{l}17.86 \\
\pm 0.11\end{array}$ \\
\hline
\end{tabular}

Ciprofloxacin was used as standard drug at $50 \mathrm{mg} / \mathrm{ml}$. All the values are in Mean \pm S.D $(\mathrm{n}=3)$. Statistical analysis of data was carried out by one-way ANOVA. 
Table 2- Antifungal activity of compounds.

\begin{tabular}{|c|c|c|c|c|c|c|}
\hline \multirow[b]{3}{*}{$\begin{array}{c}\text { Compound } \\
\text { No. }\end{array}$} & \multicolumn{6}{|c|}{ Diameter of zone of inhibition (mm) } \\
\hline & \multicolumn{3}{|c|}{ Candida albicans } & \multicolumn{3}{|c|}{ Aspergillus niger } \\
\hline & $25 \mathrm{mg} \mathrm{ml}^{-1}$ & $50 \mathrm{mg} \mathrm{ml}^{-1}$ & $100 \mathrm{mg} \mathrm{ml}^{-1}$ & $25 \mathrm{mg} \mathrm{ml}^{-1}$ & $50 \mathrm{mg} \mathrm{ml}^{-1}$ & $100 \mathrm{mg} \mathrm{ml}^{-1}$ \\
\hline 2 & $7.16 \pm 0.15$ & $12.5 \pm 0.10$ & $19.33 \pm 0.11$ & $6.66 \pm 0.11$ & $11.76 \pm 0.05$ & $18.16 \pm 0.20$ \\
\hline $3 \mathbf{a}$ & $9.46 \pm 0.30$ & $15.7 \pm 0.26$ & $21.13 \pm 0.05$ & $8.93 \pm 0.05$ & $15.36 \pm 0.05$ & $20.16 \pm 0.20$ \\
\hline $3 b$ & $10.5 \pm 0.10$ & $16.53 \pm 0.31$ & $22.7 \pm 0.10$ & $10.33 \pm 0.41$ & $15.63 \pm 0.20$ & $22.16 \pm 0.20$ \\
\hline $3 \mathbf{c}$ & $9.17 \pm 0.20$ & $14.6 \pm 0.10$ & $20.7 \pm 0.26$ & $8.6 \pm 0.20$ & $13.2 \pm 0.20$ & $20.16 \pm 0.20$ \\
\hline $3 d$ & $10.3 \pm 0.10$ & $13.16 \pm 0.05$ & $21.7 \pm 0.26$ & $9.73 \pm 0.30$ & $16.36 \pm 0.20$ & $21.23 \pm 0.25$ \\
\hline $3 \mathbf{e}$ & $11.3 \pm 0.10$ & $17.6 \pm 0.20$ & $24.46 \pm 0.25$ & $11.03 \pm 0.05$ & $17.6 \pm 0.05$ & $23.8 \pm 0.20$ \\
\hline $3 f$ & $9.2 \pm 0.20$ & $14.23 \pm 0.25$ & $20.46 \pm 0.11$ & $8.7 \pm 0.26$ & $14.16 \pm 0.20$ & $20.23 \pm 0.15$ \\
\hline $5 \mathbf{a}$ & $11.13 \pm 0.15$ & $18.1 \pm 0.10$ & $24.16 \pm 0.15$ & $10.7 \pm 0.10$ & $17.2 \pm 0.20$ & $23.73 \pm 0.05$ \\
\hline $5 b$ & $10.6 \pm 0.20$ & $18.0 \pm 0.10$ & $23.1 \pm 0.10$ & $10.2 \pm 0.10$ & $16.56 \pm 0.32$ & $22.3 \pm 0.32$ \\
\hline $5 c$ & $8.63 \pm 0.20$ & $15.16 \pm 0.20$ & $21.23 \pm 0.15$ & $8.23 \pm 0.15$ & $14.3 \pm 0.26$ & $20.5 \pm 0.10$ \\
\hline $5 d$ & $10.26 \pm 0.15$ & $17.23 \pm 0.15$ & $23.53 \pm 0.46$ & $9.6 \pm 0.20$ & $16.16 \pm 0.20$ & $22.16 \pm 0.20$ \\
\hline $5 e$ & $9.4 \pm 0.20$ & $15.43 \pm 0.20$ & $22.2 \pm 0.10$ & $9.13 \pm 0.15$ & $15.63 \pm 0.20$ & $21.63 \pm 0.20$ \\
\hline Fluconazole & & $24.16 \pm 0.20$ & $31.56 \pm 0.32$ & & $23.5 \pm 0.10$ & $30.23 \pm 0.15$ \\
\hline
\end{tabular}

Fluconazole was used as standard at $50 \mathrm{mg} / \mathrm{ml}$. Data are given as Mean \pm S.D. $(\mathrm{n}=3)$. Statistical analysis of data was carried out by paired $\mathrm{t}-$ test.

\section{CONCLUSIONS}

Various 4-[\{1-(substituted)methylidine $\}$-amino]-3-(4-pyridyl)-5-mercapto-4H-1,2,4-triazol and $N$-[5-(4-substituted)-1H-1,2,3-triazol-1-yl]isonicotinamide derivatives were synthesized from isonicotinic acid hydrazide. The structure antimicrobial activity relationship of the synthesized compounds, based on the structures of the final compound, which possessed good antimicrobial activity, revealed that the introduction of an aryl moiety generally reduced the antimicrobial activity according to the nature of the aryl moiety. The antimicrobial activities including antibacterial and antifungal properties of the synthesized compounds showed a significant activity as compared with standard drugs.

\section{REFERENCES}

1. B.S. Holla, R. Golsalves, S. Shenoy, II Farmaco. 43, 574, (1998)

2. S. Ersan, S. Nacak, R. Berkem, II Farmaco. 53, 773, (1998)

3. H. Yuksek, A. Demirbas, A. Ikizler, C.B. Johansson, C. Celik, A. A. Ikizler, Arzneim. Forsch. Drug Res. 47, 405, (1997)

4. A. A. Ikizler, F. Ucar, N. Demirbas, A. Ikizler, Indian J. Heterocycl Chem. 61, 271, (1999)

5. B. Tazkoparan, N. Gokhan,, G. Aktay, E. Yesilada, M. Ertan, Eur. J. Med Chem. 34, 743, (2000)

6. G. T. Zitouni, Z. A. Kaplancikli, K. Erol, F. Kilic. II Farmaco. 54. 218, (1999)

7. N. Demirbas, D. Ugurluoglu, Bioorg. Med. Chem. 10,3717, (2002)

8. H. Emilsson, H. Salendar, J. Gaarder, Eur. J. Med. Chem. 21, 333, (1985)

9. M. Kritsanida, A. Mouroutsou, P. Marakos, N. Pouli, S. P. Garoufalias, C. Pannecouque, M. Witvouw, E. D. Clercq, II Farmaco. 57, 253, (2002)
10. X. Collin, A. Sauleu, J.Coulon, Bioorg. Med. Chem. 13, 2601, (2003)

11. Y. A. Al-Soud, M. N. Al-Dweri, N. A. Al-Masoudi, II Farmaco. 59, 775, (2004)

12. J. K. Makrandi, P. Kumar, J. Mohan, Ind. J. Chem., 46B, 1883, (2007)

13. M. G. Mamolo, E. Banfi, IL Farmaco, 51, 71, (2001)

14. V. Mathew, J. Keshavayya, V. P. Vaidya, Eur. J. Med. Chem., 41, 1048, (2006)

15. P. Molina, A. Lorengo, J. Elguero, Tetrahedron Lett., 25, 5427, (1984)

16. J. V. Bennett, J. L. Brodie, E. J. Benner, W. M. M. Kirby, Simplified, accurate method for antibiotic assay of clinical specimens. Appl. Microbiol. 14: 170, (1966)

17. P. R. Murray, E. J. Baron, M. A. Pfaller, F. C. Tenover, R. H. Yolken, In Manual of Clinical Microbiology, G. L. Wood, J. A. Washington, Eds. Am. Soc. Microbiol. Washington, DC, 1995

18. R. T. Vasileff, L. Vrang, S. L. West, H. Zhang, X. X. Zhou, Antimicrob. Agents Chemother., 39, 1329 (1995)

19. I. H. Siddique, K. I. Loken, H. H. Hoyt, Increase in sensitivity for the assay of neomycin in milk. Appl. Microbiol. 13:635, (1965)

20. National Committee for Clinical Laboratory Standards (NCCLS), Approved standard document M-7A, Villanova, PA, 1985.

\section{ACKNOWLEDGEMENTS}

The authors are thankful to University Grant Commission, New Delhi for providing financial assistance in the form of JRF. Thanks are due to Jamia Hamdard, IIT-Delhi and IIT-Roorkee for scanning the NMR spectra, Faculty of science Jamia hamdard for IR Spectra and CDRI (Central Drug Research Institute) Lucknow. 